\title{
Exploring the robustness of insect-inspired visual navigation for flying robots
}

\author{
Alex Dewar, Paul Graham, Thomas Nowotny and Andrew Philippides \\ Centre for Computational Neuroscience and Robotics, University of Sussex, Brighton, UK \\ andrewop@sussex.ac.uk
}

\begin{abstract}
Having previously developed and tested insect-inspired visual navigation algorithms for ground-based agents, we here investigate their robustness when applied to agents moving in three dimensions, to assess if they are applicable to both flying insects and robots, focusing on the impact and potential utility of changes in height. We first demonstrate that a robot implementing a route navigation algorithm can successfully navigate a route through an indoor environment at a variety of heights, even using images saved at different heights. We show that that in our environments, the efficacy of route navigation is increased with increasing height and also, for those environments, that there is better transfer of information when using images learnt at a high height to navigate when flying lower, than the other way around. This suggests that there is perhaps an adaptive value to the storing and use of views from increased height. To assess the limits to this result, we show that it is possible for a ground-based robot to recover the correct heading when using goal images stored from the perspective of a quadcopter. Through the robustness of this bio-inspired algorithm, we thus demonstrate the benefits of the ALife approach.
\end{abstract}

\section{Introduction}

View-based homing (Cartwright and Collett, 1983; Graham and Wystrach, 2018) is a mode of navigation that is seen across a wide range of species (Wang and Spelke, 2002; Collett et al. 2013). It involves an animal storing visual information about how the world appears so that future navigation can be guided by comparison between the animal's current view and the stored information. Among animals, social insects, such as ants, bees and wasps, are often hailed as champion navigators (Collett et al., 2013) as they learn to navigate complex routes after a single trial with limited computational resources (e.g. brains of ca $1 \mathrm{M}$ neurons). They achieve this remarkable feat as they have evolved for this task and so have developed specialised eyes, brains and behaviours, meaning they provide ample inspiration for engineers seeking to develop low-cost solutions (Graham and Philippides, 2017). This mode of navigation represents an efficient computational solution because of the direct relationship between positions in the world and the perception of the world from those positions. Specifically, in real-world environments, views define unique locations and orientations and furthermore change predictably with translation and rotation (Zeil et al., 2003, Wystrach and Graham, 2012). In combination, these properties mean that views can be used for navigation in elegant ways which has directly led to a variety of biomimetic visual navigation algorithms (Möller and Vardy, 2006). Inspired by the route navigation of ants, we have developed a view-based homing algorithm capable of route navigation through simulated natural habitats with routes showing many characteristics of ant routes (Baddeley et al., 2012, Philippides et al., 2015) and which has been successfully tested on wheeled robots in several indoor and outdoor environments (Baddeley et al. 2011, Kodzhabashev and Mangan, 2015, Knight et al., 2019).

The question therefore arises as to whether the algorithm can generalise to the habitat and behaviour of flying insects such as bees. The brains of bees and ants - which are closely related hymenopterans - show many conserved features and their workers face a similar foraging task. Therefore, we might expect the same navigation algorithm to work both on the ground and in the air. If so, it would not only shed light on the neural computations necessary for visual learning in insects, but would also provide a base for further study of the importance of different eye structures and specialised navigation behaviours (notably learning walks versus learning flights, (Zeil et al., 1996, Nicholson et al., 1999)). One key issue to be overcome is that both flying insects and airborne robots experience natural variation in height driven by, for instance, wind conditions, rather than their desired movements.

Here we therefore test the ability of our algorithm to generalise across variation in flight heights. We use a high precision gantry robot in an indoor environment to show that route navigation with our algorithm is possible for a robot operating at varying heights. We additionally show how such information could feasibly be used to transfer learning from a UAV to a ground-based robot. Note that whilst the simulations described in this article are open loop, the algorithms described are computationally simple enough to 
run on a typical robot in real time Knight et al. (2019).

\section{Route navigation with a gantry robot}

To assess how our algorithm is affected by changing height, we utilised our robot gantry system ${ }^{1}$ The robot gantry comprises a panoramic camera mounted on a frame that can move to any point within a $2.8 \times 1.6 \times 1.2 \mathrm{~m}$ arena. This provides more realistic images than could be obtained in a virtual environment (i.e. subject to natural changes in lighting etc.), whilst still allowing for precise control of the camera's position, including, crucially, its height - something which could not be achieved with an outdoor robot. As our intention is to use these algorithms outdoors (Philippides et al. 2016) the gantry arena was filled with plastic foliage (mostly grasses and ferns, but also some flowers) between 300 and $800 \mathrm{~mm}$ in height and of varying widths, as naturalistic landmarks (Fig. 1). Fifteen plants were placed into three polystyrene blocks in a horseshoe shape in the centre of the arena (black polygons in Fig. 2A-B) and largely filled these regions. The polystyrene blocks were $\sim 50 \mathrm{~mm}$ in height so we took our lowest transect for analysis at $150 \mathrm{~mm}$. The walls of the gantry are white plastic and so provide a homogeneous background. The camera produces RGB images of $720 \times 60$ pixels covering $360^{\circ}$ of azimuth and $\sim 50^{\circ}$ of elevation (extending roughly equally above and below the camera's 'horizon'). Before use, panoramic RGB images are converted to greyscale, 'unwrapped', cropped to remove visual artefacts from the camera mount, and down-sampled to $360 \times 29$ pixels, which is similar to both the resolution and field of view of an ant's eye. We have also shown previously that resolutions on this order are sufficient for good visual homing performance Wystrach et al. (2016). Using images with too high a resolution can introduce noise in the form of high-frequency spatial information, whilst too low a resolution can lead to visual aliasing.

The familiarity-based route navigation algorithm proceeds in two steps. The first step is to acquire training views or 'snapshots' at regular intervals (here $25 \mathrm{~mm}$ ) along a userdefined route (snapshots are shown as red circles in Fig.22AB). The route we report in this paper (selected to maximise length) comprised two straight lines starting at (100, 1700) and proceeding through vertices at $(900,900)$ and $(1700$, 900). Training snapshots were taken from the nearest grid points to this route (using Bresenham's algorithm; $n=82$ ) oriented so that they are parallel to the nearest line. While the number of snapshots used is somewhat arbitrary, we are more interested here in the effect of changing heights on homing performance rather than in pushing the algorithm to its limits, although a similar process was used successfully on a wheeled robot in real time Knight et al. (2019).

To recapitulate the route, the agent samples views at multiple orientations from each position and selects the most

\footnotetext{
${ }^{1}$ Note that the image databases and other data sets used in this article are available online Dewar et al. (2020).
}

A

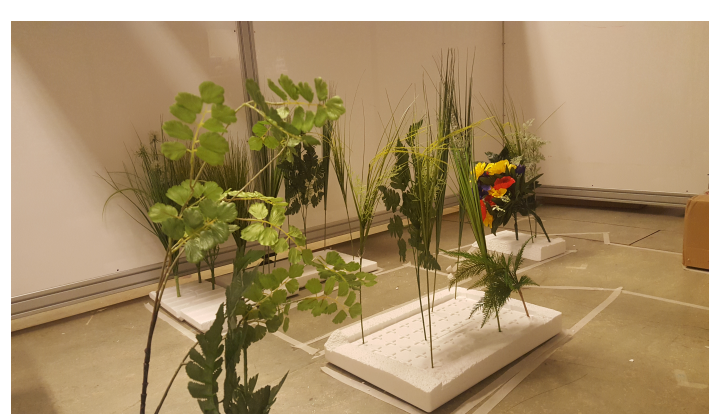

B

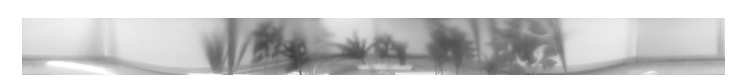

C

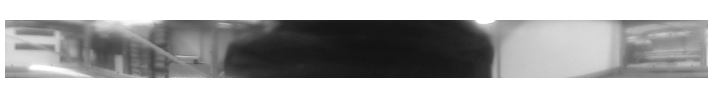

Figure 1: The robot gantry system. A: A photograph of the robot gantry with the plastic plants inside. B: A view from the gantry camera when the arena is filled with plastic plants. C: As B, but with a single, large landmark in the centre of the arena. Note that here the walls have been removed from the gantry arena, providing a view of more distal visual cues within the workshop.

familiar view by comparison either directly with the snapshots (dubbed the 'Perfect Memory' algorithm, (Baddeley et al. 2012), or by inputting the views to an artificial neural network (ANN) trained to output the familiarity of the training snapshots (discussed in more detail later). The most familiar view specifies the direction in which to move and so the agent moves a step in this direction, and then repeats the rotational sampling, picking the most familiar heading and moving another step.

To compare the accuracy of our algorithm using images from different heights, we first gathered training images from positions along the training route at one height, and then assessed what heading would have been recovered by sampling the world at a grid of positions within the arena at a different height. Specifically, images were acquired $10 \mathrm{~cm}$ apart at heights ranging from $150-650 \mathrm{~mm}$ in steps of $100 \mathrm{~mm}$. This gave a grid of $17 \times 29$ points for each height (resulting in 366 images when positions 'within' plants are excluded). As images from the training route are facing the correct direction by definition, we calculate errors in heading from each test position by taking the absolute angular difference between the computed heading and the heading of the nearest snapshot (errors capped at $90^{\circ}$ ). This is not necessarily the 'correct' heading but serves as a fair comparator across conditions. As we only expect the algorithms to function effectively from regions within a corridor around the training route, when we generate overall errors for a condition, we use image positions within $200 \mathrm{~mm}$ of the route only (green arrows in Fig. 2A-B), with distances again set 
as the distance to the nearest snapshot.

We first examine the performance of the Perfect Memory algorithm. The Perfect Memory algorithm works by comparing rotated versions of the current view with all stored snapshots across all rotations. The smallest difference between a snapshot and the current view at a given rotation indicates greatest familiarity. We thus generate Rotational Image Difference Functions (RIDFs) (Zeil et al., 2003) between each snapshot and the current view, by rotating the current view through $360^{\circ}$ in $1^{\circ}$ steps (which simulates a rotational scan of the world), and calculating the average absolute difference at each heading for each stored snapshot, yielding a 360-value RIDF for each. The heading at which we see the minimum difference across all of the RIDFs is then deemed the best matching heading. If we were recapitulating the route, the algorithm would next take a step in this best-matching direction and the process would repeat. Here we simply generate the best-matching headings from the grid of positions at different heights which allows a more direct comparison of the accuracy with which heading information can be recalled. Note that the Perfect Memory algorithm is not meant to represent the way that an insect might navigate, but does interrogate the visual information available along routes. This is because, as has been detailed previously (Zeil et al. 2003; Philippides et al., 2011), the presence of a clear minimum in an RIDF indicates that a snapshot can be used to derive an appropriate heading.

\section{Route navigation for an agent with varying flight height}

The training views (red circles in Fig. 2A-B) are taken from a manually chosen route through an arena in which we had placed plastic plants (mainly grasses and ferns which are quite homogenous as well as a few more distinctive flowers) as naturalistic features. Crucially, the stored views are oriented along the route direction and so an agent can subsequently navigate the route by recalling this 'correct' movement direction when close to the point at which the snapshot was stored. To test the accuracy of route navigation, we derived the headings from images taken at a grid of positions $10 \mathrm{~cm}$ apart within the arena acquired at multiple heights (specifically, 150, 250, 350, 450, 550 or $650 \mathrm{~mm}$ ), by comparison with sets of route snapshots also gathered from one of the set of heights (Fig. 2). In general, the algorithm is resilient to changes in height, with reliable headings produced even as the height varies, indicating that the visual information relevant for navigation can be transferred over various heights. For instance, whether using snapshots from the lowest height and comparing it to images from either the lowest or highest plane (Fig. 2A), or using snapshots from the highest height and again comparing to low and high (Fig. 2B), the headings derived (arrows in Fig. 2A-B) are generally in the correct directions, especially when close to the route (green arrows, Fig.2A-B).
Interestingly, when considering the headings derived near the route at the centre of the arena, it seems that there may be more resilience to height changes when snapshots are taken at a greater height and tested at a lower height (Fig. 2 $\mathrm{B}$, top), than vice versa (Fig. 22A, bottom). To investigate this phenomenon further, we assessed accuracy for training data from either 150 or $650 \mathrm{~mm}$, tested against data from each of the other heights (Fig. 2C-D respectively). As with the previous examples, first, there is a good transfer across all heights with relatively low median errors overall, showing the resilience of these methods to changes in height. Second, as would be expected, errors between different heights increase with increasing discrepancy. Third, there is a slight trend that these errors are slightly lower when trained with high and tested with low images, than vice versa. This can be seen in the greater spread of the data at more of the high heights when trained with $150 \mathrm{~mm}$ images (Fig. $2 \mathrm{C}$, right side), than when trained with $650 \mathrm{~mm}$ images which only have a large variation at the two lowest height planes (Fig. 2D, left side). The spread of errors is especially important because being iterative, our route navigation algorithms can typically cope with small errors in direction. Thus we also assess the percentage of positions resulting in 'wrong' headings, defined as heading errors greater than $30^{\circ}$ (other thresholds give qualitatively similar results) when trained with data at one height and tested at each of the other heights (Fig. 2E). As would be expected from the previous results, the best performance is along the diagonal, where training and test heights are similar. However, focussing on the performance for each training height (each row in Fig. $2 \mathrm{E}$ ), there is again a suggestion of better transference for the higher heights, with best overall performance at $550 \mathrm{~mm}$. The reason for this can be seen in Fig. 6, which shows the homing performance when training with a higher image and testing with a lower one (Fig. 6A) and vice versa (Fig. 6B). With snapshots recorded at greater heights the plants occupy less of the image and, accordingly, they drive the heading less than the more rotationally reliable cue: the broad shape of the arena. This low-frequency spatial information is still useful when the 'agent' is lowered to $150 \mathrm{~mm}$, however, in the low-to-high condition the rotational cue given by the plants becomes useless at higher heights where they are less visible.

\section{ANN-based route navigation for a flying robot}

We next test route navigation for images gathered at different heights using an Infomax familiarity-based neural network to learn a compact encoding of the views as in (Baddeley et al., 2012) (henceforth referred to as Infomax). In this variant, instead of remembering all the views and comparing the current and stored views on an individual basis, a single-layer neural network is trained with the stored views to learn the familiarity of the training views using an Infomax training rule (Lulham et al., 2011). The network is 
A
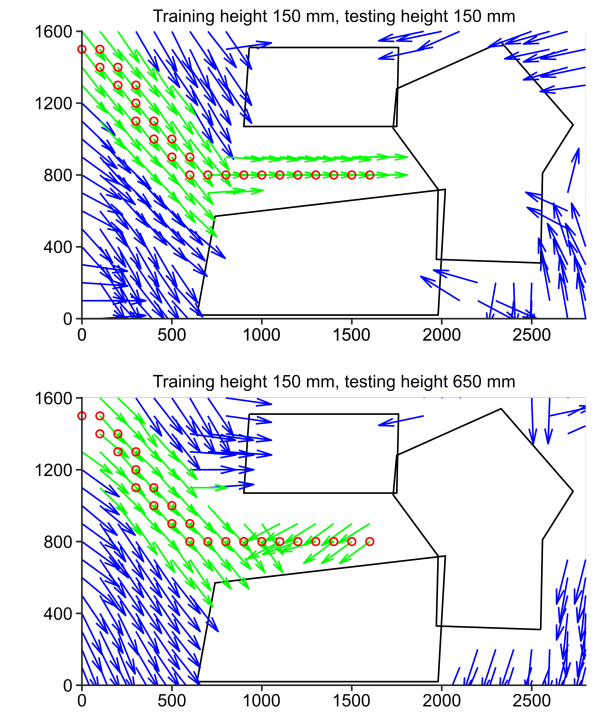

C

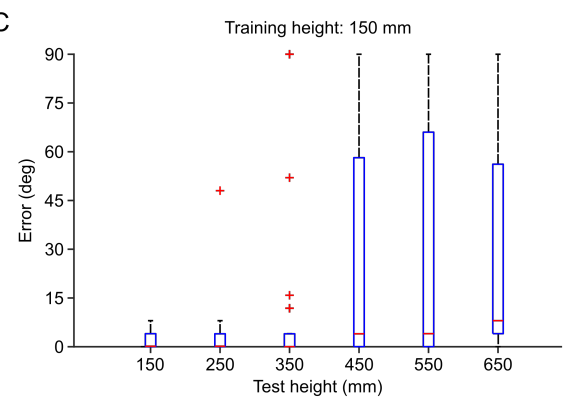

B

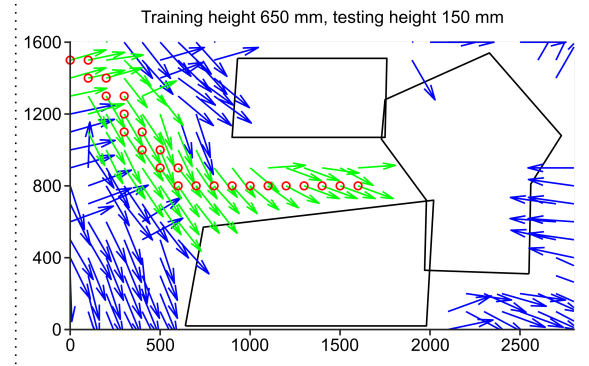

Training height $650 \mathrm{~mm}$, testing height $650 \mathrm{~mm}$

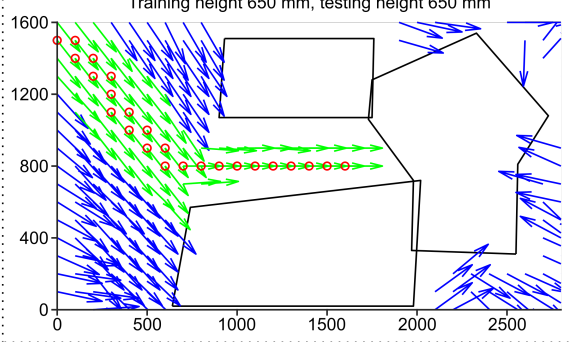

D $\quad$ Training height: $650 \mathrm{~mm}$

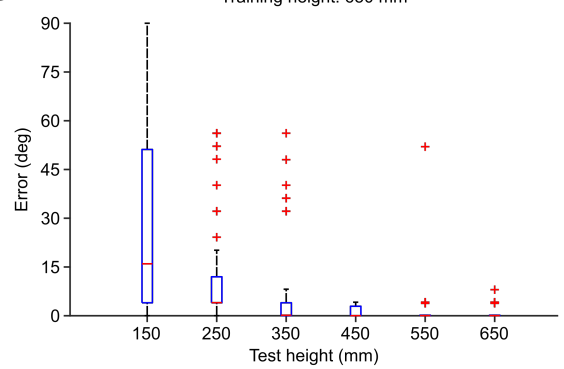

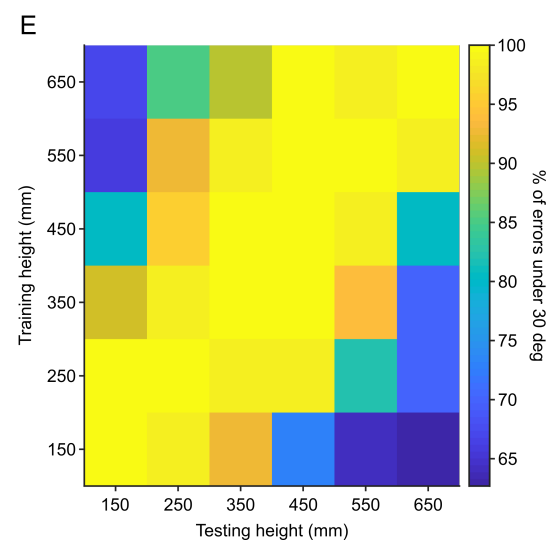

Figure 2: Route navigation algorithms are robust to changes in height. A: Arrows showing estimated headings through an arena containing plastic flowers and plants as calculated by a route navigation algorithm trained with images taken at $150 \mathrm{~mm}$ and tested at a height of $150 \mathrm{~mm}$ (top) or $650 \mathrm{~mm}$ (bottom). Training views, or 'snapshots', were taken at positions shown with red circles proceeding from top-left of the arena to the centre in two straight lines, with 'correct' heading of the snapshots being aligned with the training route (see methods). Positions close to the route are indicated with green arrows; this corresponds to the 'error corridor' used for panel C. Black polygons show areas containing plastic flowers and plants. B: Estimated headings for route navigation algorithm with snapshots taken at $650 \mathrm{~mm}$ and tested at $150 \mathrm{~mm}$ (top) and $650 \mathrm{~mm}$ (bottom). Symbols as in A. C-D: Distribution of errors in estimated headings when trained with snapshots at $150 \mathrm{~mm}(\mathrm{C})$ and $650 \mathrm{~mm}$ (D) tested at a range of heights, shown on the $x$-axis. Red lines indicate medians; boxes cover 25 th and 75 th percentiles; whiskers extend to 1.5 times the inter-quartile range; red crosses show outliers outside whiskers. E: Percentage of the 'error corridor' that has an error under $30^{\circ}$ for each test height ( $x$-axis) when snapshots are taken from each training height ( $y$-axis). Yellow shows higher percentage correct, blue lower (see colour bar for scale). 
then presented with the current view at a range of rotations and the orientation which yields the smallest activation (and thus the greatest familiarity) is taken as the best-guess heading. For this test we placed a number of cardboard boxes of different heights $(\min =9.8 \mathrm{~cm} ; \max =60.4 \mathrm{~cm})$ within the arena to provide visual stimuli. Additionally, the walls of the arena were removed so more distal visual cues were available in the form of the visual panorama of the workshop in which the gantry is housed. First we acquired the stored views for an arbitrary route within the arena at a separation of $2.5 \mathrm{~cm}$ ( $n=60$; indicated by red crosses in Fig. 3 ). We then calculated the best-guess headings for the Perfect Memory and Infomax algorithms (Fig. 3A and B, respectively) for images collected from the gantry at a range of $x, y$ and $z$ coordinates. As well as being more biologically plausible than the Perfect Memory variant of the algorithm, using a neural network-based approach also has the advantage that the time taken to estimate a heading is constant with respect to the number of training images, which becomes especially important for longer routes.

Fig. 3 shows that robust performance is given by both the Perfect Memory and Infomax flavours of the visual homing algorithm. Although performance is understandably poorer for locations where the original training route is obscured by boxes, nonetheless, the algorithms mostly yield headings parallel to the training route, indicating that there is sufficient distal visual information (in the workshop) to drive reliable homing. Moreover, performance does not decay substantially with changes in height, which suggests that even quite dramatic amounts of visual noise in a real-world environment would be unlikely to lead the agent astray. The quality of performance is notable for the Infomax case, where the only data required in memory is a $644 \times 644$ matrix of weights, as opposed to the entire cache of stored views $(58 \times 720 \times 60$ pixels in total $)$ and, unlike the latter, does not increase with the number of stored views. Accordingly, the time taken to compute a heading is also considerably faster for Infomax.

\section{Can images from a quadcopter be used by a ground-based robot to recover a heading?}

To assess how different both heights and perspectives could be between training and test views, we next compared images gathered from a quadcopter with a wheeled robot. In this iteration of our research, it was not technically possible to test the full route navigation algorithm, However, as the algorithm relies, in its heart, on deriving a heading from an RIDF comparison, here we see if accurate headings can be derived in this way as a first step.

The dataset was gathered in an open section of field located near the Sussex University campus in Preston Park. The transect covered $84 \mathrm{~m}$ in $3 \mathrm{~m}$ intervals and started from an open field and progressed towards a nearby tree line. This location thus combined areas where elements making up the skyline were both distant and near, the former being easier for visual homing algorithms (Stürzl and Zeil, 2007; Wystrach et al., 2016). Panoramic images were collected using a Kodak PixPro SP360 camera at 3 m intervals along the transect at a height of $40 \mathrm{~cm}$. Heights were kept consistent and the camera level through the use of a tripod with an in-built spirit level.

Aerial data was collected from a GoPro Hero 3 attached to a DJI Phantom 2 quadcopter. The quadcopter was flown manually at three heights, 3,5 and $8 \mathrm{~m}$, by an experienced pilot attempting to follow the transect used to acquire the panoramic images. In order to do this, the pilot started the drone slightly before the start position of the panoramic data, and headed towards the same goal tree. There was necessarily variation both in 3-D position and attitude during data acquisition, but this was accepted as we aim to see if visual homing algorithms are tolerant of this type of error. Videos of $\sim 10$ seconds covered the 84 metre distance. Frames were extracted from these videos at regular intervals between visually determined start and end points so that each frame was $\sim 3 \mathrm{~m}$ apart. This leads to 28 stills for the 3 and $5 \mathrm{~m}$ heights, but the $8 \mathrm{~m}$ height had only 26 as the drone started to descend before the end of the transect.

Because of visual aliasing, there is little success in place recognition when routes and goals are at different heights (as judged by comparing images and seeing if the best matching image is at the same location, data not shown). However, in (Philippides et al. 2016) we saw that when height varies, the region over which an image can be used to recover directional information is larger than the region over which place recognition is possible. This previous work compared images from $40 \mathrm{~cm}$ to $2 \mathrm{~m}$ so we wanted to assess if images from a UAV could be used to guide a ground-based robot.

We therefore gathered panoramic images from a height of $40 \mathrm{~cm}$ and used these as a test route to compare with images from a quadcopter. We first made the sky a homogeneous colour (by thresholding on the blue channel at a value of 170) as the light gradient can provide a very strong, but spurious cue, by which homing algorithms can gain information. Both images were then resized so that pixels covered the same azimuth and elevation. For the drone images, this meant reducing the amount of ground in the images, which we determined by making sure the median value of the height of the skyline was the same across the two sets of images. We then extracted slices of the same 'width' of image from the ground-based panoramic view as was captured in the forward-facing quadcopter image (Fig. 4A), with each slice 'facing' in a different angle. While ground-based and quadcopter images were taken from similar positions and at similar headings, alignment was not perfect so we have picked what we feel is the 'correct' heading (i.e. when this image is best aligned with the UAV image) by eye (red vertical lines in Fig $4 \mathrm{~A}$ ).

To analyse if the information would transfer, we exam- 
A


B
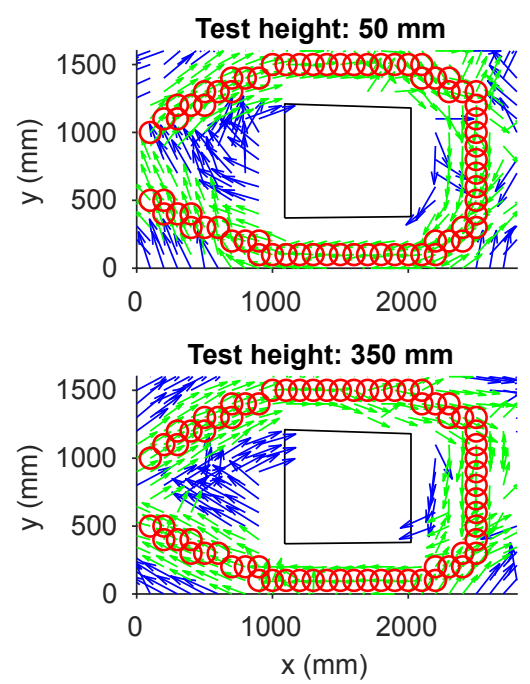

Perfect memory
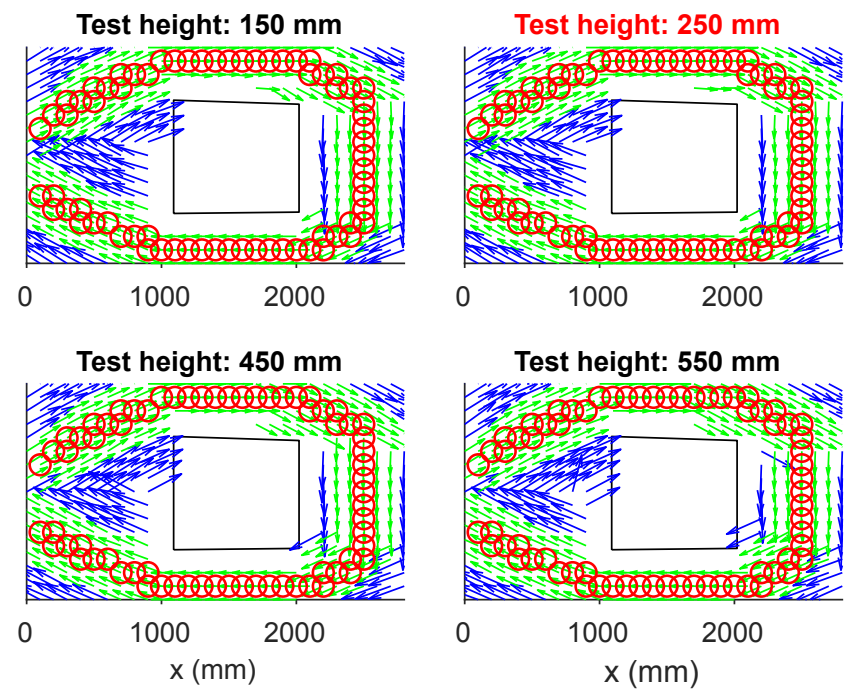

Infomax
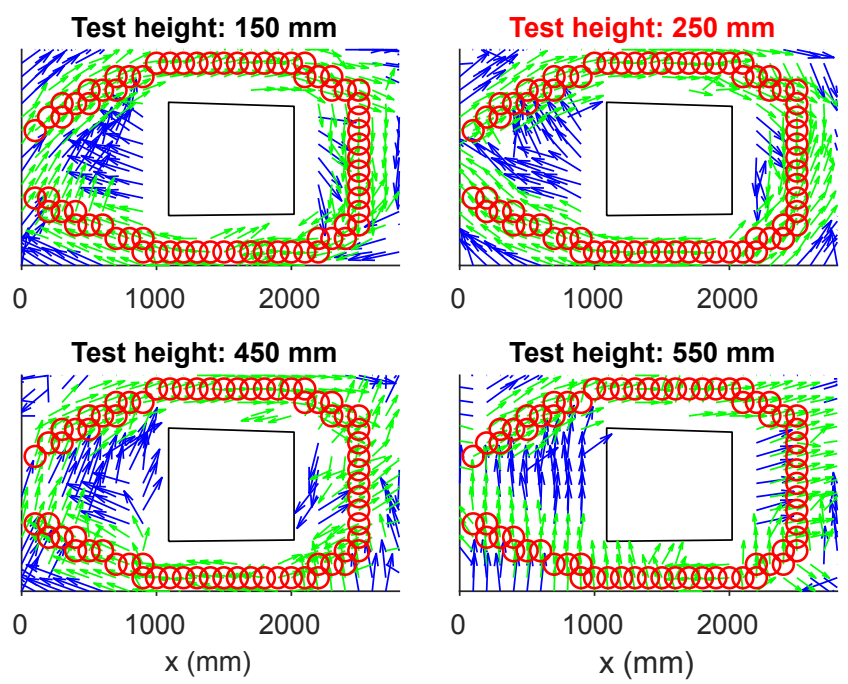

Figure 3: Estimated headings for different homing algorithms at a range of heights using the robot gantry. Training views, or snapshots, were recorded at a height of $250 \mathrm{~mm}$ (red circles) with image resolution of $7 \times 90$ pixels. The heading direction was the direction between consecutive circles and the route proceeded clockwise from the upper open end around the large object (black box). A: Best-guess headings using Perfect Memory from other positions and heights are shown by arrows. The height of the test positions is in the panel title. B: Best-guess headings using Infomax algorithm. Symbols as for A. 
A
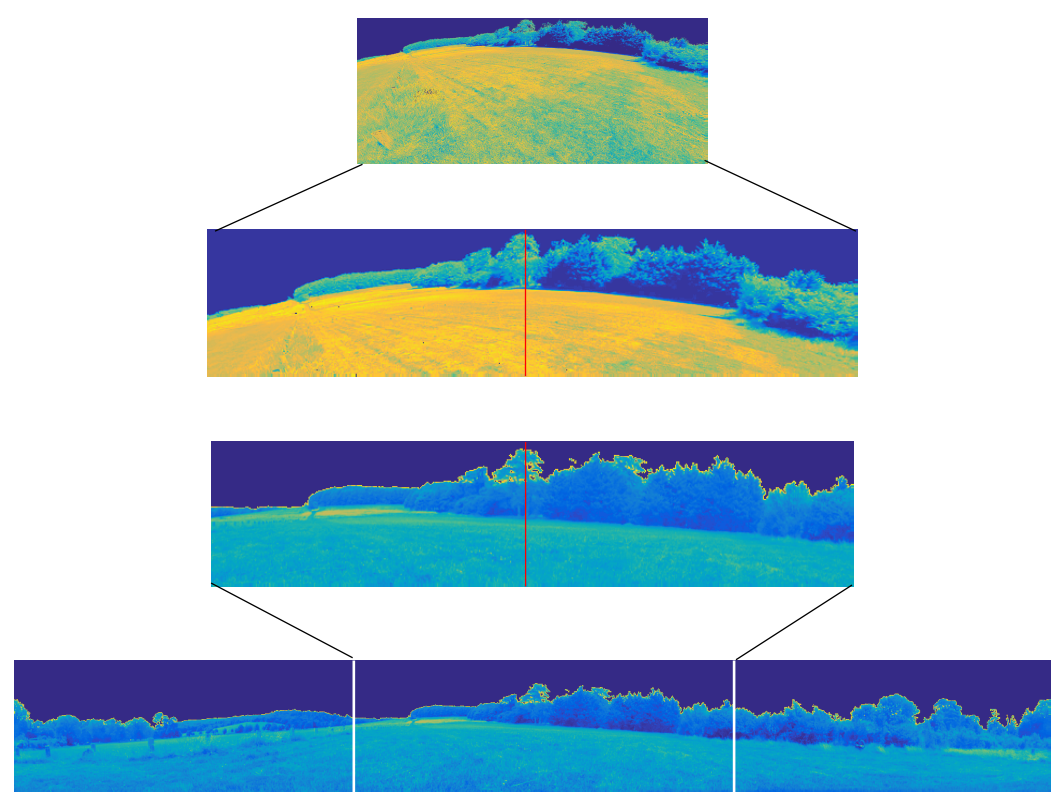

B
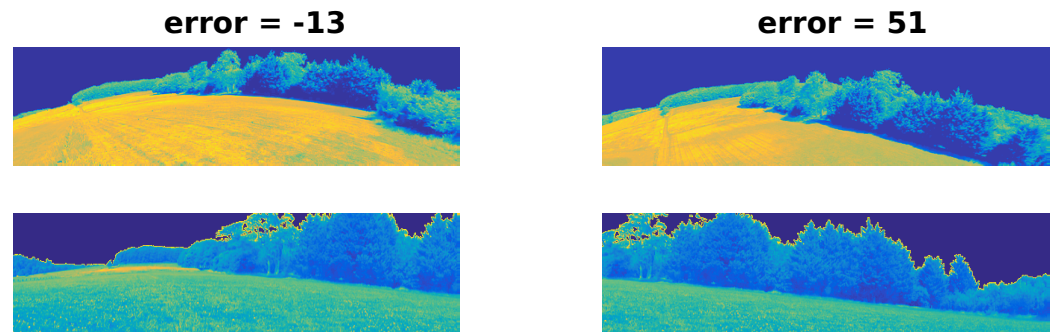

Figure 4: Pre-processing and comparison of images from flying and wheeled robots. A: Processing images from a forwardfacing camera on a UAV (top panel) so that it can be compared to a portion of a panoramic image from a wheeled robot (bottom panel; white lines show the angular range in azimuth covered by the forward-facing camera). Both images have been converted to greyscale from RGB (via rgb2gray function in MATLAB) and have had the sky set to a uniform value of 0 , as described in the text. Both images are cropped and resized (imresize function in MATLAB) so that they cover the same angular range in elevation (see text) and azimuth and have the same number of pixels, as displayed in the central panels. To find the bestmatching heading, the image from the UAV is compared to different azimuthal windows (white lines in bottom panel) from the wheeled robot, which simulates rotating the image, with the best-matching heading being determined by the minimum RMS pixel difference across rotations. B: Example images from a UAV at $3 \mathrm{~m}$ (left) and at $8 \mathrm{~m}$ (right) with the best-matching image part from the wheeled robot across all rotations and route images.

A

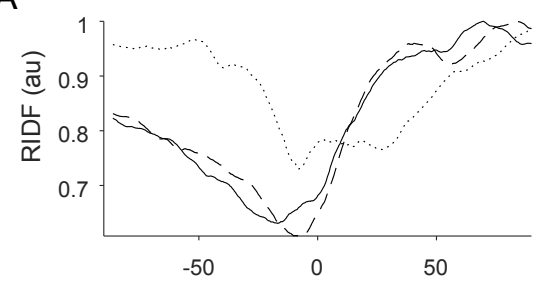

B

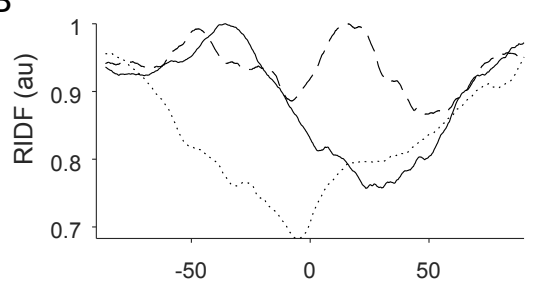

C

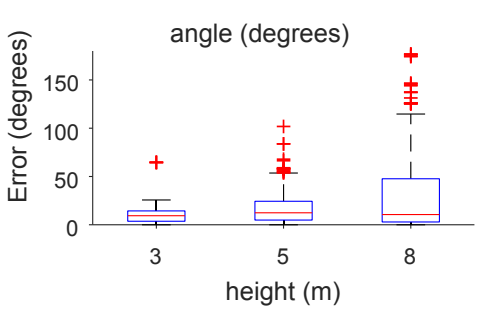

Figure 5: Matching a ground-based route with a goal from a UAV. A, B: Typical RIDFs for a route at $3 \mathrm{~m}$ and $8 \mathrm{~m}$, respectively. $\mathrm{C}$ : Error in the predicted bearing across all goal positions and all test positions for routes at 3,5 and $8 \mathrm{~m}$ (see labels on $x$-axis). Red lines show median, whole boxes cover the 25 th and 75 th percentile and whiskers extend to 1.5 times the inter-quartile range. Red pluses are outliers outside this range. 

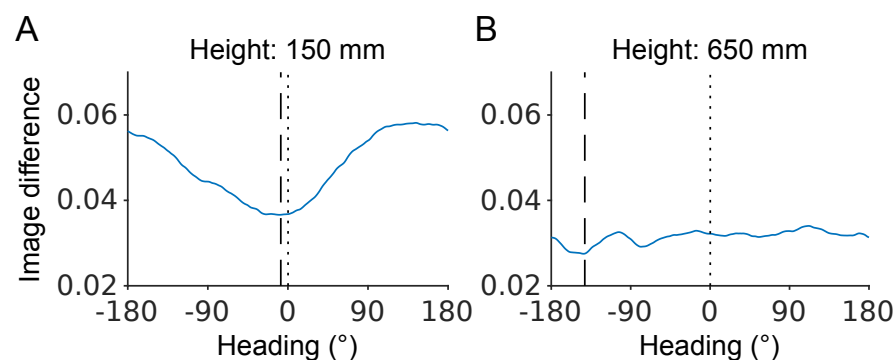

C



E

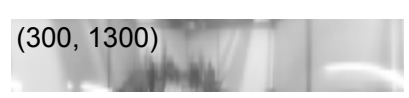

D

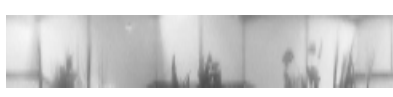

$\mathrm{F}$

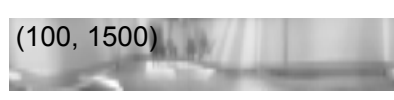

Figure 6: Example test locations for the Perfect Memory trials in the plants environment, showing that training images from a higher height can be used effectively at a lower height, but not vice versa. A: An RIDF comparing a training image recorded at $(1600,800,650)$ with a testing image at the same $x$ and $y$ location but a lower height: (1600, 800, 150). The dotted line indicates the goal direction and the dashed line the best-guess heading given by the RIDF; in this case the estimated heading is reliable. B: As for A, but with the heights of the training and testing images inverted. Note that in this case, the RIDF is shallower and the error on the best-guess heading is much higher. C, D: The 'current' (i.e. testing) views at $(1600,800,150)$ and $(1600,800,650)$, respectively. E, F: The best-matching snapshots for the two conditions as selected by the Perfect Memory algorithm $(x / y$ coordinates inset).

ined headings derived from RIDFs between UAV images and the panoramic ground-based views. Typical RIDFs are shown for matching three goal images (from start, middle and end of the route) with quadcoptor views at heights of 3 and $8 \mathrm{~m}$ (Fig. 5A-B). Unsurprisingly, the $3 \mathrm{~m}$ views match better with the ground-based robot, with minima near to the correct heading, but there is considerable variation depending on the goal location. Comparing the errors in heading across all possible goal positions (Fig. $5 \mathrm{C}$ ), the general pattern holds true with the $3 \mathrm{~m}$ route generating lower errors. However, because of the difficulties in place recognition, the best-matching view from the quadcopter is not necessarily close to the goal location. Thus had the route flown been curved rather than straight, the errors could have been much greater. These results should therefore be treated with caution but give us optimism that our route navigation algorithms could transfer from flying to ground robots despite very different visual perspectives.

\section{Conclusions}

To demonstrate the feasibility of our biomimetic visual navigation algorithm, we showed that route navigation can function effectively despite differences in the height of the training route compared to the route being navigated, whether images are simply stored or used to train an ANN to generate a compact route encoding. This reinforces the work of (Zeil et al., 2003, Philippides et al., 2016, Murray and Zeil, 2017) which showed that the utility of single images for visual homing, increases with increasing height. In addition, we showed that visual information from single images can transfer between wheeled and flying robots, despite changes in tilt during flight and a forward facing camera. This lends promise to our attempts to transfer route information between different robot platforms, which has implications in, for instance, search and rescue operations where different robots could be used for exploration and retrieval. The use of a holistic route memory, which is easy to transfer between robots, will aid this endeavour and is the subject of ongoing work. Additionally, it would be interesting to see, for instance, how well tilt-invariant encodings, or encodings based on the height of objects above the horizon, would work, an avenue we are currently investigating. However, much more work needs to be undertaken to prove this performance in closed-loop systems outdoors.

In addition, our results show that bees could use the same navigation strategies as ants, despite the fact that their flight height may vary from trial to trial. The consequence of learning a set of views that are displaced from a goal and oriented towards the goal, but from a range of different directions, is that the insect develops a large catchment area over which learnt views can guide the individual back to the goal (Wystrach et al., 2013, Dewar et al., 2017). Could the same be true of height? In simple terms, views from multiple heights would turn a catchment area into a catchment volume (Lihoreau et al., 2016, Murray and Zeil, 2017). The results from our modelling suggest that, not only is the information gained at one height useful at another height, but it might be possible to increase the utility of learnt information by active modulation of height both in learning and during recall. For instance, as views stored higher have better transfer to other heights, increasing height when lost is a sensible strategy - something we would like to test. In summary, by mimicking biology in a situated and embodied model we have shown that a navigation algorithm based on the behaviour of ants is suitable for both flying robots and insects, thus showing the benefits of the artificial life ethos for engineering and biology.

\section{Acknowledgements}

Authors were funded by EPSRC grants EP/P006094/1 and EP/S030964/1. 


\section{References}

Baddeley, B., Graham, P., Husbands, P., and Philippides, A. (2012). A model of ant route navigation driven by scene familiarity. PLoS Computational Biology, 8:1.

Baddeley, B., Graham, P., Philippides, A., and Husbands, P. (2011). Holistic visual encoding of ant-like routes: Navigation without waypoints. Adaptive Behavior, 19(1):3-15.

Cartwright, B. A. and Collett, T. S. (1983). Landmark learning in bees - experiments and models. Journal of Comparative Physiology, 151:521-543.

Collett, M., Chittka, L., and Collett, T. S. (2013). Spatial memory in insect navigation. Current Biology, 23(17):R789-R800.

Dewar, A., Graham, P., Nowotny, T., and Philippides, A. (2020). Data set for alife paper. http:// placeholder.for.doi.

Dewar, A. D. M., Wystrach, A., Philippides, A., and Graham, P. (2017). Neural coding in the visual system of Drosophila melanogaster: How do small neural populations support visually guided behaviours? PLoS Computational Biology, 13(10):e1005735.

Graham, P. and Philippides, A. (2017). Vision for navigation: What can we learn from ants? Arthropod Structure and Development, 46(5):718-722.

Graham, P. and Wystrach, A. (2018). View-based homing. In Shackelford, T., editor, Vonk J. Encyclopedia of Animal Cognition and Behavior. Springer, Cham.

Knight, J., Sakhapov, D., Domcsek, N., Dewar, A., Nowotny, T., Graham, P., and Philippides, A. (2019). Insect-inspired visual navigation on-board an autonomous robot: Real-world routes encoded in a single layer network. In Press, I., editor, Proceedings of Alife 19.

Kodzhabashev, A. and Mangan, M. (2015). Route following without scanning. In Biomimetic and Biohybrid Systems, . International Publishing, pages 199-210.

Lihoreau, M., Ings, T. C., Chittka, L., and Reynolds, A. M. (2016). Signatures of a globally optimal searching strategy in the three-dimensional foraging flights of bumblebees. Scientific Reports, 6(1):30401.

Lulham, A., Bogacz, R., Vogt, S., and Brown, M. W. (2011). An Infomax algorithm can perform both familiarity discrimination and feature extraction in a single network. Neural Computation, 23(4):909-926.
Möller, R. and Vardy, A. (2006). Local visual homing by matched-filter descent in image distances. Biological Cybernetics, 95:413-430.

Murray, T. and Zeil, J. (2017). Quantifying navigational information: The catchment volumes of panoramic snapshots in outdoor scenes. PLOS ONE, 12:10.

Nicholson, D. J., Judd, S. P. D., Cartwright, B. A., and Collett, T. S. (1999). Learning walks and landmark guidance in wood ants (Formica rufa). Journal of Experimental Biology, 202(13):1831-1838.

Philippides, A., Baddeley, B., Cheng, K., and Graham, P. (2011). How might ants use panoramic views for route navigation? Journal of Experimental Biology, 214:445-451.

Philippides, A., Graham, P., Baddeley, B., and Husbands, P. (2015). Using neural networks to understand the information that guides behavior: a case study in visual navigation. artificial neural networks. pages 227-244.

Philippides, A., Steadman, N., Dewar, A., Walker, C., and Graham, P. (2016). Insect-inspired visual navigation for flying robots. In Publishing, S. I., editor, Conference on Biomimetic and Biohybrid Systems, pages 263274.

Stürzl, W. and Zeil, J. (2007). Depth, contrast and viewbased homing in outdoor scenes. Biological Cybernetics, 96:519-531.

Wang, R. F. and Spelke, E. S. (2002). Human spatial representation: Insights from animals. Trends in Cognitive Sciences, 6(9):376-382.

Wystrach, A., Dewar, A., Philippides, A., and Graham, P. (2016). How do field of view and resolution affect the information content of panoramic scenes for visual navigation? A computational investigation. Journal of Comparative Physiology A, 202(2):87-95.

Wystrach, A. and Graham, P. (2012). What can we learn from studies of insect navigation? Animal Behaviour, 84(1):13-20.

Wystrach, A., Mangan, M., Philippides, A., and Graham, P. (2013). Snapshots in ants? New interpretations of paradigmatic experiments. Journal of Experimental Biology, 216(10):1766-1770.

Zeil, J., Hofmann, M., and Chahl, J. (2003). Catchment areas of panoramic snapshots in outdoor scenes. Journal of the Optical Society of America A, Optics and Image Science, 20:450-469. 
Zeil, J., Kelber, A., and Voss, R. (1996). Structure and function of learning flights in ground-nesting bees and wasps. Journal of Experimental Biology, 199(1):245252. 